\title{
Whole Spontaneous Spinal Epidural Hematoma
}

\author{
Kyeong-Wook Yoon ${ }^{1}$, Jae Gyok Song ${ }^{2}$, Jae-Wook Ryu ${ }^{3}$, Young-Jin Kim ${ }^{1}$ \\ ${ }^{1}$ Department of Neurosurgery, Dankook University College of Medicine, Cheonan, Korea \\ ${ }^{2}$ Department of Anesthesiology and Pain Medicine, Dankook University College of Medicine, Cheonan, Korea \\ ${ }^{3}$ Department of Thoracic and Cardiovascular Surgery, Dankook University College of Medicine, Cheonan, Korea
}

A 26-year-old male who had no underlying disease, including coagulopathy, underwent thoracotomy and bleeding control due to hemothorax. On the fifth postoperative day, paralysis of both lower limbs occurred. Urgent spine magnetic resonance imaging showed a massive anterior spinal epidural hematoma from C2 to L1 level with different signal intensities, which was suspected to be staged hemorrhage. Hematoma evacuation with decompressive laminectomy was performed. The patient's neurologic deterioration was recovered immediately, and he was discharged without neurological deficits. A drug history of naftazone, which could induce a druginduced platelet dysfunction, was revealed retrospectively. To our knowledge, this is the first report of whole spontaneous spinal epidural hematoma in a young patient, with a history of hemorrhoid medication.

Keywords: Epidural hematoma; Naftazone; Spinal hematoma

\section{Introduction}

Spontaneous spinal epidural hematoma (SSEH) is a rare entity with unclear etiology. Precipitating factors described in previous reports include anticoagulation therapy for cardiac valves, hemophilia, factor XI deficiency, long-time aspirin use, drug abuse, and vascular malformation [1]. SSEH of unknown origin compromises approximately $40 \%$ of SSEH cases in the literature [2]. According to several reports, SSEH is expressed in all ages, with a male to female ratio of 1.4:1 [3]. Most case reports have demonstrated SSEH involving a short vertebral column. They have suggested several hypotheses regarding its pathogenesis without citing clear evidence. We report the first case of whole SSEH in a young adult, who has taken naftazone for hemorrhoid treatment. The patient underwent rapid surgical decompression and achieved full recovery without neurologic deficits.

\section{Case Report}

A 26-year-old male was scheduled for wedge resection of bullae and bleeding control for left hemothorax after chest tube insertion for spontaneous pneumothorax. His medical history did not show any significant abnormality. Physical examination and laboratory findings were unremarkable.

He was referred to our emergency department for the treatment of hemopneumothorax with massive bleeding, which occurred after the chest tube insertion. Upon arrival, approximately $400 \mathrm{~mL}$ of bloody fluid was drained from the chest drainage tube. His initial hemoglobin $(\mathrm{Hb})$ was $11.0 \mathrm{mg} / \mathrm{dL}$. Two units of packed red blood cells (PRC) were infused to treat hypovolemic tachycardia (95 beats/min), with mild hypotension (95/50 mm Hg). Initial prothrombin time/international normalized ratio, activated partial thromboplastin time and platelet count

Received Jun 20, 2013; Revised Aug 21, 2013; Accepted Sep 12, 2013

Corresponding author: Young-Jin Kim

Department of Neurosurgery, Dankook University College of Medicine,

201 Manghyang-ro, Dongnam-gu, Cheonan 330-715, Korea

Tel: +82-41-550-3979, Fax: +82-41-552-6870, E-mail: spine1225@naver.com 
were 13.0/1.12, 24.7 seconds, and $245,000 / \mathrm{mm}^{3}$, respectively. The patient's bleeding time was not checked.

Two hours later, laboratory findings revealed $\mathrm{Hb} 10.5$, hematocrit (Hct) 30.4 , and platelet count $220,000 \mathrm{~mm}^{3}$. One unit of PRC and three units of fresh frozen plasma (FFP) were infused. The patient was transferred to the operating room after normalization of the abnormal laboratory findings.

Bullectomy and hematoma evacuation were performed. Bleeding control of three small arteries on the disrupted pleural tag on the apex of the left lung was conducted under general anesthesia. The operation took two hours. The total infused fluids during the operation were crystalloid $900 \mathrm{~mL}$, colloids $400 \mathrm{~mL}$, and PRC 2 units. The patient's $\mathrm{Hb}$ level was $11.6 \mathrm{mg} / \mathrm{dL}$, and the platelet count was $175,000 / \mathrm{mm}^{3}$, immediate postoperatively.

On the second postoperative day, the patient complained of a headache; and oral acetaminophen 1,300 mg was given two times a day. On the third postoperative day, the patient complained of a headache, dizziness, and nausea during ambulation. On the fifth postoperative day, the patient complained of motor weakness of lower extremities, which was determined as grade 4/5. Hypesthesia was detected below the T3 dermatome, and deep tendon reflexes of knee joint were increased bilaterally. Immediate spine magnetic resonance imaging (MRI) revealed a massive anterior spinal epidural hematoma, compressing the spinal cord from C2 to L1 vertebrae (Fig. 1). Hematoma evacuation with decompressive total laminectomy was performed at T 2,3,6, 7, 8 and left partial hemi-laminectomy at T9, 10. Hypesthesia was presented below the T2 dermatome, with T4- 6 being most vulnerable to ischemia. Cervical laminectomy was not necessary, as motor grade of upper extremities was normal. On the microscopic operative field, there was no definite bleeding focus. Intraoperative lab findings were unremarkable. We conducted a platelet function test with platelet function analyser (PFA)-100 to rule out platelet dysfunction and found platelet dysfunction (collagen/epinephrine, more than 300 seconds [normal range, 61-192 seconds], collagen/adenosine di-phosphate [ADP], 124 seconds [normal range, 61-110 seconds]).

In the three hours of operation, we transfused 10 units of platelet concentrates, 9 units of FFP, and 5 units of PRC. Motor grade was recovered to 5/5 in both lower limbs, with a remaining tingling sensation after the operation. On the second postoperative day, the patient's motor and sensory functions returned to normal in both limbs and the trunk. On the eighth postoperative day, the PFA value decreased to the normal range. One month after the operation, the patient was discharged without neurologic deficits. Postoperative MR image was obtained two months after surgery and showed total evacuation of hemorrhage (Fig. 2).

\section{Discussion}

SSEH is concerned with several underlying diseases, and is correlated with many predisposing factors including organic vascular disease, hemodialysis, coagulation disorder, and anticoagulation therapy [4]. The incidence of SSEH is $1 / 1,000,000$, and less than $1 \%$ consists of spinal epidural space occupied lesions [5]. A few reports have stated that SSEH comes from the epidural venous plexus in the spinal epidural space. However, Miyagi et al. [1] have raised an objection that arterial bleeding is more convincing for SSEH, because pressure of the venous

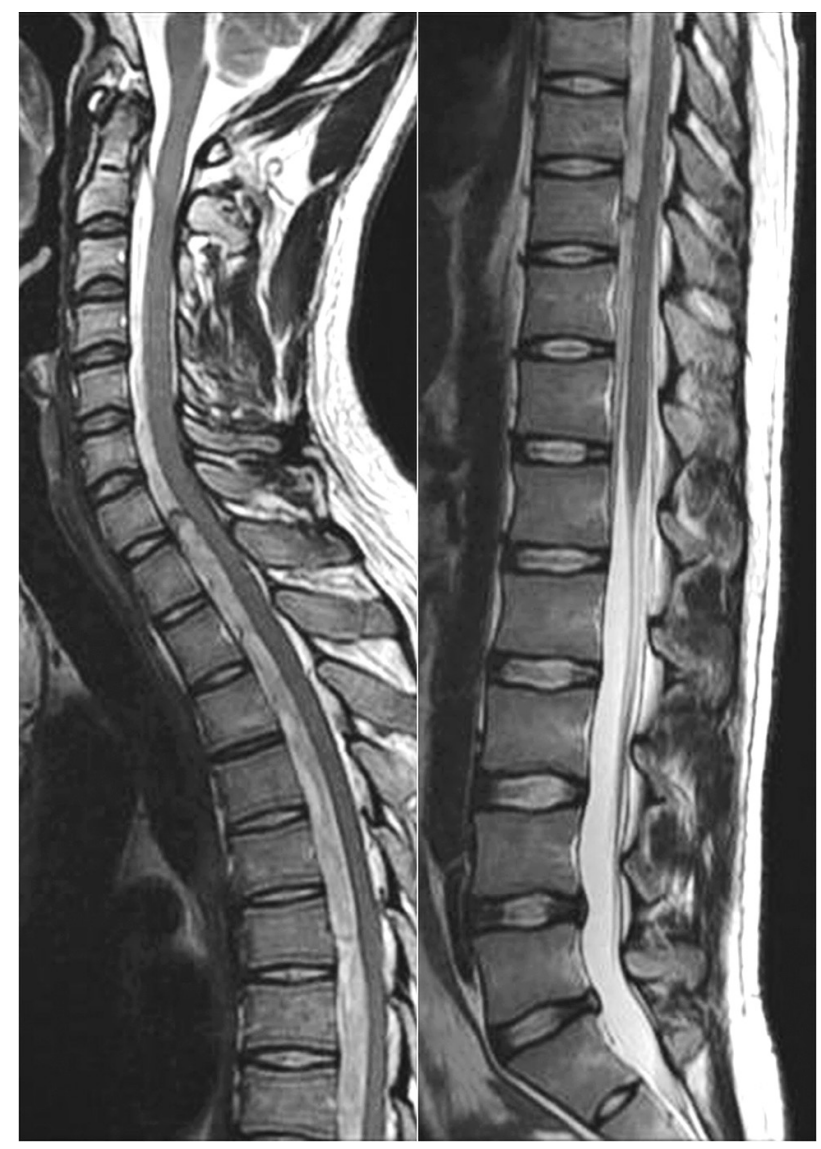

Fig. 1. Preoperative sagittal T2 weighted magnetic resonance images showing a massive epidural hemorrhage from C2 to L1 level. 
plexus is lower than that of the spinal epidural space. The initial general symptoms are neck or back pain and neurologic deficits, such as motor weakness or rapid progression of hypesthesia, which intimates a quick formation of epidural hematoma and cord compression. Also, the fact that arterial bleeding causes SSEH is another supporting background.

In the present case, the opinion of a thoracic surgeon was that the bleeding arteries in the ruptured pleural tag were too small to make such a massive hematoma. Our patient had no definite underlying disease that could cause SSEH; however, we noticed that the patient had received medication (naftazone) for hemorrhoid for seven days up to the day before admission. Naftazone (Mediaven, 1, 2-naphthoquinone-2-semicarbazone) is used to treat mild to moderate peripheral vascular diseases or venous insufficiency (varicose veins, hemorrhoids). Naftazone can increase vascular endothelial cell proliferation and inhibit platelet aggregation induced by ADP or thrombin in-vitro, together with thrombin stimulating 5-hydroxytryptamine release. This patient had taken 30 $\mathrm{mg}$ of naftazone for seven days. Previous ex-vivo and invivo studies have shown that naftazone has similar inhibi-

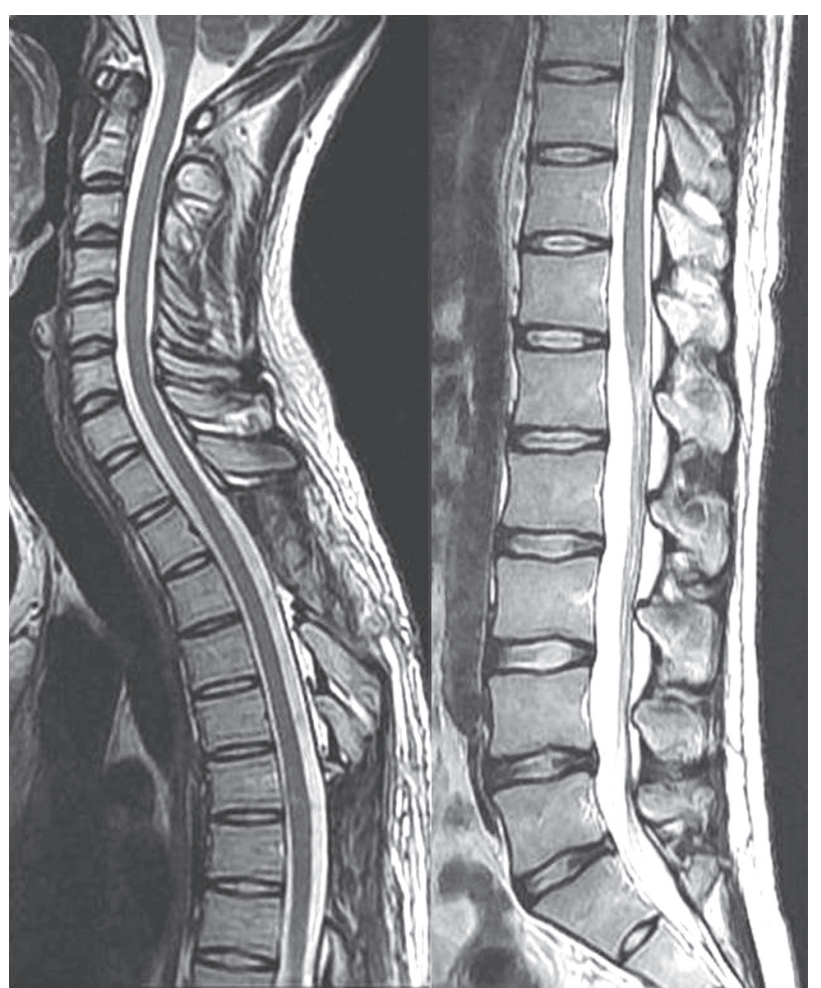

Fig. 2. Postoperative magnetic resonance images show total evacuation of epidural hemorrhage. tory effects on rat platelet functions as ticlopidine [6]. However, there has been no report of complications following naftazone therapy and the duration of persisting antiplatelet effect after discontinuation.

After thoracotomy and bleeding control due to hemothorax, the patient was started on mefenamic acid $250 \mathrm{mg}$, three times a day, for four days. On the fourth postoperative day, the patient was treated with intravenous ketorolac $30 \mathrm{mg}$, three times a day. The patient was diagnosed with SSEH the following day. Non-aspirin non-steroidal anti-inflammatory drug (NSAID) for pain control after operation generally causes a transient, dosedependent and modest increase of bleeding time. However, these prolongations often do not exceed the upper limit of the normal range for bleeding time [7]. However, if NSAIDs are used with other anticoagulants (wafarin, aspirin, other NSAIDs, herbal medications), its adverse effects on coagulation can be dangerous $[7,8]$.

Abnormal PFA finding was recovered after platelet concentrate infusion and discontinuation of NSAIDs. It was certain that the interaction of the above several drugs had induced platelet dysfunction, although the dose of each drug (naftazone, mefenamic acid and ketorolac) alone was not enough to cause significant bleeding. We concluded that the drugs may have additive or synergistic antiplatelet effect.

Presently a significant number of people take medications, herbal medicines or foods that can decrease platelet function, without informed awareness [8]. Hence it is suggested to confirm normal platelet function before conducting neuroaxial anesthesia. For patients taking anticoagulants before surgery, the type of drug should be identified even if preoperative laboratory findings show no abnormality. We tested the present patient's platelet function using a PFA-100 instrument. This device appears to be superior to measuring bleeding time, and is recommended as a replacement screening test [9]. However, PFA-100 is sensitive to many variables that influence platelet function, including platelet number, Hct, drugs, platelet receptor defects and Von Willebrand's factor defect. In this case, the patient's laboratory findings were all within normal limits, including Von Willebrand's factor, and the patient had no family history of bleeding tendency.

In our patient, we transfused several pints of PRC and FFP to correct the abnormal lab findings. This is considered a factor in platelet dysfunction as well. Massive 
transfusion may induce coagulopathy in several aspects [10].

To our knowledge, a long-segment SSEH (occurring from $\mathrm{C} 2$ to $\mathrm{L} 1$ ) has never been reported. The reason for the formation of such SSEH, covering the whole spine, is obscure. In more serious coagulopathy, SSEH is formed over three or four segments. Our case of mild coagulopathy does not explain this phenomenon. It is very important that prompt surgical decompression is performed for neurologic recovery and that anticoagulation therapy be temporarily halted, even if patient demands it. Conservative treatment is recommended only if neurologic deficits resolve spontaneously soon after the onset or in cases without neurologic deficits.

\section{Conflict of Interest}

No potential conflict of interest relevant to this article was reported.

\section{References}

1. Miyagi Y, Miyazono M, Kamikaseda K. Spinal epidural vascular malformation presenting in association with a spontaneously resolved acute epidural hema- toma. Case report. J Neurosurg 1998;88:909-11.

2. Penar PL, Fischer DK, Goodrich I, Bloomgarden GM, Robinson F. Spontaneous spinal epidural hematoma. Int Surg 1987;72:218-21.

3. Ravid S, Schneider S, Maytal J. Spontaneous spinal epidural hematoma: an uncommon presentation of a rare disease. Childs Nerv Syst 2002;18:345-7.

4. Deger SM, Emmez H, Bahadirli K, et al. A spontaneous spinal epidural hematoma in a hemodialysis patient: a rare entity. Intern Med 2009;48:2115-8.

5. Groen RJ, Ponssen H. The spontaneous spinal epidural hematoma. A study of the etiology. J Neurol Sci 1990; 98:121-38.

6. Durand P, Bloy C, Peltier-Pujol F, Blache D. In-vitro and ex-vivo inhibition of blood platelet aggregation by naftazone. J Pharm Pharmacol 1996;48:566-72.

7. Schafer AI. Effects of nonsteroidal antiinflammatory drugs on platelet function and systemic hemostasis. J Clin Pharmacol 1995;35:209-19.

8. Abebe W. Herbal medication: potential for adverse interactions with analgesic drugs. J Clin Pharm Ther 2002;27:391-401.

9. Harrison P. Platelet function analysis. Blood Rev 2005;19:111-23.

10. Elmer J, Wilcox SR, Raja AS. Massive transfusion in traumatic shock. J Emerg Med 2013;44:829-38. 